\title{
Superior parietal lobule: a role in relative localization of multiple different elements
}

\author{
A.Vialatte $^{1}$, Y. Yeshurun ${ }^{2}$, A. Z. Khan ${ }^{3}$, R. Rosenholtz ${ }^{4}$, L. Pisella ${ }^{1}$ \\ Affiliations:
}

${ }^{1}$ Integrative Multisensory Perception Action \& Cognition Team (ImpAct), INSERM U1028, CNRS UMR5292, Lyon Neuroscience Research Center (CRNL), Lyon, France; University of Lyon 1, Lyon, France; Hospices Civils de Lyon, Mouvement \& Handicap, Neuro-Immersion Platforms, Lyon, France

${ }^{2}$ Psychology Department, University of Haifa, Haifa, Israel

${ }^{3}$ School of Optometry, University of Montreal, Montreal, Canada

4 Department of Brain \& Cognitive Sciences, Massachusetts Institute of Technology, Cambridge, MA USA

Corresponding Author:

L. Pisella PhD,

16 avenue du doyen Lépine

69676 Bron,

0472913405 ,

laure.pisella@inserm.fr 


\section{ABSTRACT (197 words)}

Simultanagnosia is an impairment in processing multiple visual elements simultaneously consecutive to bilateral posterior parietal damage, and neuroimaging data have specifically implicated the superior parietal lobule (SPL) in multiple-element processing. We previously reported that a patient with focal and bilateral lesions of the SPL performed slower than controls in visual search but only for stimuli consisting of separable lines. Here we further explored this patient's visual processing of plain object (colored disk) versus object consisting of separable lines (letter), presented in isolation (single object) versus in triplets. Identification of objects was normal in isolation, but dropped to chance level when surrounded by distracters, irrespective of eccentricity and spacing. We speculate that this poor performance reflects a deficit in processing objects' relative locations within the triplet (for colored disks), aggravated by a deficit in processing the relative location of each separable line (for letters). Confirming this, performance improved when the patient just had to detect the presence of a specific colored disk within the triplets (visual search instruction), while the inability to identify the middle letter was alleviated when the distracters were identical letters that could be grouped, thereby reducing the number of ways individual lines could be bound.

Keywords : crowding - object individuation - posterior parietal cortex - visual perception simultanagnosia 


\section{INTRODUCTION}

Vision is a sense that allows us to apprehend a relatively large space in one glance, and to process in parallel, at least to some degree, the multiple objects lying in this space as well as their spatial relationship. We constantly face complex visual scenes and we have to process multiple elements simultaneously (e.g., leaf, trunk, branch) in order to ascertain a global meaning (e.g., a tree, a forest). Consecutive to extensive bilateral occipito-parietal damage patients exhibit "an extreme restriction of visual attention, such that only one object is seen at a time" (Bálint 1909; Husain \& Stein 1988). Later, Wolpert coined the term "simultanagnosia" for these patients (Wolpert 1924).

More recent studies of patients with more focal lesions (or using voxel-based morphometry in patients with diffuse posterior cortical atrophy) have refined the neural substrate underlying simultanagnosia: the superior parietal lobule (SPL) is specifically involved (Khan et al. 2016; Neitzel et al. 2016; Valdois et al. 2019). These studies and others (e.g. Luria 1959; Nacchache et al 2000; Jackson et al. 2006; Demeyere et al 2008; Thomas et al. 2012; Chechlacz et al. 2015; Strappini et al. 2017) have also further questioned the level (perceptual or attentional) and the underlying neuro-cognitive mechanisms possibly causing simultanagnosia. For example, Luria (1959) observed that his patient reported only one shape when two different simple shapes were presented simultaneously, but shape perception was facilitated when the two presented shapes were identical. Based on this modulation of the patient's performance by perceptual grouping, Luria (1959) suggested a deficit at the attentional level (for the relationships between attention and perceptual organization processes, see Kimchi et al. 2007; Yeshurun et al. 2009). Alternatively, Neitzel et al (2016) showed that simultaneous visual processing ability correlated with visual short-term memory performance and visual processing speed in patients with diffuse posterior cortical atrophy. The present paper pursues this line of research aimed at elucidating the nature of simultanagnosia and the role of SPL in vision.

Simultaneous visual processing is particularly necessary for reading, and the literature on simultanagnosia is often referred to in the literature of developmental dyslexia (Bogon et al. 2017; Valdois et al. 2019). A subset of dyslexic children show a reduced letter span at the Global Report task: shown a string of letters flashed in central fixation, they can report fewer letters than age-matched controls, whereas they can identify a single letter as fast as normal readers (Bosse et al. 2007; Lassus-Sangosse et al. 2008; but see Bogon et al. 2014). Neuroimaging studies (Peyrin et al. 2011, 2012; Lobier et al. 2012, 2014; Reilhac et al. 2013) 
have shown bilateral SPL hypoactivation in these developmental dyslexics compared to normal readers. In normal readers, SPL activation increases bilaterally when a visual categorization task is performed on multiple objects simultaneously compared to when the same task is performed on a single object. Suggested by these correlational results, the causal link between SPL activation and the ability to recognize multiple objects in a single fixation has been recently established thanks to the study of patient IG, a rare patient with focal and bilateral acquired lesion of the SPL. In the Global Report task with 5 letters presented simultaneously in central fixation for $200 \mathrm{~ms}$, patient IG also showed a reduced letter span (Valdois et al. 2019). In a control task in which individual letters were rapidly flashed alone serially for $200 \mathrm{~ms}$ in central vision, patient IG performed successfully. Taken into consideration with other tasks presented in Valdois et al (2019), this ruled out the possibility of a general slowing of visual perception or of reduced visual short-term memory capacity following focal bilateral SPL lesion (contrary to other patients' reports in the literature: Neitzel et al. 2016).

The performance consecutive to bilateral SPL lesions resembles developmental dyslexics' performance, not only in the Global Report task, but also in visual search. Khan et al (2016) presented to patient IG several versions of classical visual search arrays where a single target is presented among identical distracters for an unlimited duration (until detection). The time required to find the target was measured. Patient IG performed a pop-out visual search for a unique shape (find a red disk among red squares), as well as a shape and color conjunction search (find a red disk among red squares and green disks) as fast as controls. This preservation of parallel multiple object processing in visual search could be due to the fact that patient IG could perceptually "group" the identical distracters, while in the Global Report task strings of five different consonants were presented. However, she performed more slowly than controls in simple feature-present (e.g. the simplest task required to find $\mathrm{Q}$ among $\mathrm{O}$ ) and feature-absent (e.g. the simplest task required to find $\mathrm{O}$ among $\mathrm{Q}$ ) visual searches, even though she could similarly "group" the distracters. It was concluded from patient IG's performance that bilateral damage to the SPL impairs simultaneous visual processing specifically in condition requiring spatial integration of separable lines. This specific visual search deficit for figures made of multiple lines ("multifeatured shapes") had previously been reported for poor readers (Casco and Prunetti 1996); e.g. the authors showed that poor readers were as fast as controls to find a single tilted line among vertical or horizontal ones, but difficulties arose when this unique tilted line of the visual display was spatially combined with others to make a symbol. Like patient IG, poor readers showed no deficit in searches for simple features or conjunction of features 
like color and geometrical shapes. Khan et al (2016) further demonstrated that this slow search performance for visual displays involving combined separable lines in patient IG corresponded to a reduced visuo-attentional field that could be mimicked in healthy controls by a reduced visible window whose size depends on the visual complexity of the stimuli but not on the number of distracters falling in this window. The impairment of simultaneous visual processing of patient IG was therefore attributed to a reduction of the size of the spotlight of attention.

Intriguingly, several aspects of this phenomenology have parallels with visual crowding. Crowding refers to difficulty performing visual tasks in the presence of clutter, e.g. reduced ability to recognize an object when it is surrounded by distracters, in comparison to when the object appears in isolation. Crowding has been implicated in visual search difficulty; difficulty identifying a crowded peripheral target predicts difficulty for pop-out, conjunction, and featurepresent vs. feature-absent search (Rosenholtz et al. 2012). Furthermore, crowding has been described in terms of an "uncrowded window" (Pelli and Tillman 2008), the size of which depends on the complexity of individual items (Hulleman and Olivers 2017).

In the Global Report task, patient IG reported the unflanked letters (the first and last ones) but rarely the middle ones (flanked by other letters) but this reduced letter span was independent of spacing (Valdois et al. 2019). In crowding, too, observers can more readily report the first and last items in a peripheral string than the letters in the middle of the string (Bouma 1970). Crowding mainly occurs in peripheral vision but also in central vision, the crucial parameter is the inter-stimuli distance - as this distance increases performance increases, and when this distance is large enough (larger than the 'critical distance'), the distracters no longer affect performance (Bouma 1970; Toet and Levi 1992; Pelli, Palomares and Majaj 2004). Nonetheless, the connections between crowding and simultaneous visual processing following focal bilateral SPL damage might suggest that a lesion of the SPL could produce a form of excessive crowding. A common or related mechanism for crowding and clinical vision has been previously proposed for amblyopics (Greenwood et al. 2012), for dyslexics (Martelli et al. 2009) and for patients with occipito-temporal damage (ventral visual stream, Strappini et al. 2017; Sand et al. 2018).

The present study will explore this hypothesis for patients with damage of the dorsal visual stream by exploring in more depth the performance of patient IG in crowding tasks. We examined performance in identifying a crowded target in both central and peripheral vision, and in particular examined the extent to which patient IG's performance remains immune to 
the spacing between target and distracters. Furthermore, crowding causes loss of location information; for simple objects, this can lead to reporting a distracter instead of the target (Strasburger 2005; Van den Berg et al. 2012), while for more complex figures made up of multiple lines, this loss can cause a jumbling of features between items (Chang and Rosenholtz 2016). Here, by varying the task (identification of an item at a specific middle location vs. visual search) and the type of stimuli (colored disks vs letters) we examine loss of location information in patient IG. Finally, a number of researchers have also reported relief from crowding when the two items flanking the target are similar to each other but dissimilar from the target (Livne and Sagi 2007; Saarela et al. 2009; Freeman et al. 2012). We examine the effects of flanking the target with identical distracters for patient IG.

\section{METHODS}

\section{Participants}

Patient IG was 45 at the time of this experiment. When she was 29 , she suffered from an ischemic stroke related to acute vasospastic angiopathy in the posterior cerebral arteries, established with an angiogram. Magnetic resonance imaging revealed a hyperintense signal on T2 sequencing that was fairly symmetrically located at the upper and lateral occipito-parietal junction, cortically and subcortically. Reconstruction of the lesion (Pisella et al. 2000; Khan et al. 2016) indicated that it involved mainly the intraparietal and the parieto-occipital sulci with full damage to Brodmann's area 7 in the SPL, but there was also limited damage to the dorsallateral part of Brodmann's areas 19 and 18 and to the upper part of the angular gyrus (BA39) in the inferior parietal lobule (Figure 1). Patient IG presents with bilateral optic ataxia which remains chronic but never presented with either hemispatial neglect or oculomotor apraxia. Her eye movements were completely normal at clinical assessment, with latencies and amplitudes similar to controls within the functional saccadic range: Gaveau et al. (2008), for example, found deficits only for very far eccentricities, $20^{\circ}$ or greater. Such saccade amplitudes are almost never used in everyday life as they would normally be implemented with an accompanying head movement. Patient IG has a quadrantanopia in the right bottom quadrant of the visual field of both eyes, due to subcortical damage of the optic radiations below the SPL in the left hemisphere. Visual stimuli will thus be presented slightly above the ocular fixation in central vision, and in the left visual field in peripheral vision. She also presents with mild chronic simultanagnosia. Initially, her simultanagnosia was so severe that it prevented her from 
perceiving two disks presented simultaneously, but this recovered quickly (see Pisella et al. 2000). During the acute phase, she reported that she could not clearly see more than one finger of her hand at once; she is now able to see "almost" all fingers simultaneously. For the Navon test (Navon 1977), she did not show the classical global precedence effect of healthy controls: she was not slower in identifying the local letter when it was incongruent with the global letter $(\mathrm{sH}, \mathrm{hS})$ compared with when it was congruent $(\mathrm{sS}, \mathrm{hH})$. Note that for most simultanagnosic cases reported in the literature, patients tend to have larger lesions that are progressive (e.g., neurodegenerative disease such as posterior cortical atrophy), and tend to be older, with ages of around 50 and up (Gilchrist et al. 1996; Clavagnier et al. 2006; Dalrymple et al. 2007; Huberle et al. 2010; Thomas et al. 2012), and therefore tend to have more severe symptoms (e.g., cannot report the global letter in the Navon test at all) including associated symptoms such as aphasia, alexia, neglect, constructional, or oculomotor apraxia. In contrast, patient IG's performance on tests of intellectual efficiency, general executive and attentional functions, language, motor and visual abilities was normal (Valdois et al. 2019).

We also tested 4 age-matched control participants, 3 females and one male (mean age $=41.75$ $+/$ - 12.66, age range $=28-58$ ). They had no history of neurological or psychiatric disorders and they had normal vision at the experimental distance.

All experimental procedures were approved by the French health research ethics committee (CPP Nord-Ouest I, Lyon, 2017-A02562-51)

\section{Apparatus}

Participants sat in a semi-dark room with their eyes at a distance of $80 \mathrm{~cm}$ from a high-speed CRT monitor (dimensions: $40 \times 30 \mathrm{~cm}, 800 \times 600$ pixels, refresh rate: $160 \mathrm{~Hz}$ ), with their forehead and chin stabilized. Stimuli were presented on the screen using a real-time stimuli presentation (Visual Stimulus Generator ViSaGe, Cambridge Research System, Rochester, UK) along with custom-written code developed in the laboratory. Eye position was recorded using a high-speed video Eyetracker (Cambridge Research System) at $1000 \mathrm{~Hz}$. Subjects responded using a $\mathrm{ViSaGe}$ response box.

Stimuli and Procedure 
The size of the stimuli was $0.8^{\circ}$ of visual angle presented on a white background (R255, G255, B255). In the Color conditions, disks of 3 possible colors could be presented: RGB, blue (R118, G118, B148), red (R148, G118, B118), green (R118, G148, B118); we used also grey disks (R120, G120, B120) in Experiment 3. In the Letter conditions, 3 possible letters could be presented: F, N, T; we also used the letter $\mathrm{X}$ in Experiment 3.

In all tasks, a trial was composed of a 400ms fixation cross, followed by stimuli presentation for $90 \mathrm{~ms}$ (except in the variant of Task 2 in which target duration varied). Next a response screen was presented that remained until the participant pressed the button (Figure 2). Throughout each trial, we asked the participant to keep fixating the central cross. This was controlled on-line by the eye tracker (if the eyes moved, the trial was aborted and replayed at the end of the block).

Experiment 1: Is there a critical spacing in central or peripheral vision?

Tasks

Patient IG and the four age-matched control participants ran three tasks in Experiment 1, in which they had to respond whether the target was "red", "blue" or "green" in the color conditions and "F", " $\mathrm{N}$ " or " $\mathrm{T}$ " in the letter conditions by pressing three different buttons (forced choice, chance level was 1/3).

Single target presentation task

This task consisted of presenting one of the 3 possible targets (letter or color in different blocks) by itself (without distracters) at different eccentricities $\left(0^{\circ}, 3^{\circ}, 7^{\circ}\right.$ and $\left.10^{\circ}\right)$. The goal of this task was to ensure that each participant was able to distinguish isolated colors or letters at different visual eccentricities. In each block, each of the three possible targets was presented 10 times at the 4 eccentricities, distributed pseudo-randomly. Two blocks, one for letter and one for the color condition, were performed, one at the beginning and one at the end of the experiment. There were a total of 240 trials for each letter and color condition.

Central vision crowding task 
This task consisted of presenting the target in central vision $\left(0^{\circ}\right)$, for $90 \mathrm{~ms}$, flanked by 2 stimuli with target-distracters spacing of $0.8^{\circ}, 1.5^{\circ}$ and $3^{\circ}$. The distracters had the same three possible colors or letters as the target. The task was to identify the middle target. One of the distracters was the same as the target in $28 \%$ of the trials to avoid inferring the target identity from the distracters alone. Each of the 3 possible targets was presented 14 times at the 3 different spacing, distributed pseudo-randomly. There was a total of 126 trials each for the letter and color conditions. In separate blocks, we also varied the duration of stimuli presentation $(90 \mathrm{~ms}, 110$ $\mathrm{ms}, 130 \mathrm{~ms}, 150 \mathrm{~ms}$ ) while spacing remained fixed at $0.8^{\circ}$. Each of the 3 possible targets was presented 18 times at the 4 different durations, distributed pseudo-randomly. There were a total of 216 trials each for the letter and color conditions.

Peripheral vision crowding task

This task consisted of presenting one of the 3 possible targets (letter or color in different blocks) in peripheral vision at different eccentricities $\left(3^{\circ}, 7^{\circ}, 10^{\circ}\right)$ and with different spacing (targetdistracter center-to-center distance) as follows:

For the letter condition:

$\circ$ Eccentricity $3^{\circ}: 0.8^{\circ}, 1^{\circ}, 1.5^{\circ}, 2^{\circ}, 2.5^{\circ}$

$\circ$ Eccentricity $7^{\circ}: 1^{\circ}, 1.5^{\circ}, 2.5^{\circ}, 3.5^{\circ}, 5^{\circ}, 6^{\circ}$

$\circ$ Eccentricity $10^{\circ}: 2^{\circ}, 3^{\circ}, 4^{\circ}, 5^{\circ}, 6^{\circ}, 7^{\circ}$

For the color condition:

$\circ$ Eccentricity $3^{\circ}: 0.8^{\circ}, 1^{\circ}, 1.5^{\circ}, 2^{\circ}, 2.5^{\circ}$

$\circ$ Eccentricity $7^{\circ}: 0.9^{\circ}, 1^{\circ}, 1.5^{\circ}, 2^{\circ}, 3.5^{\circ}, 5^{\circ}$

$\circ$ Eccentricity $10^{\circ}: 1^{\circ}, 1.5^{\circ}, 2^{\circ}, 3.5^{\circ}, 5^{\circ}, 6^{\circ}$

For example, for the $3 \mathrm{deg}$ eccentricity with 0.8 spacing, the distracters would appear at $2.2 \mathrm{deg}$ and at 3.8 deg aligned horizontally. The participant's task was to identify the middle target. Each of the 3 target colors (or letters) was presented 14 times at the 3 peripheral eccentricities and at the different spacings described above, distributed pseudo-randomly. The distracters had the same three possible colors or letters as the target. One of the distracters was similar to the 
target in $28 \%$ of the trials to avoid inferring the target identity solely from the identification of the two distracters. Overall, there were a total of 966 trials each for the letter and color conditions. Breaks were offered every 161 trials.

Procedure and Data analysis

The central vision crowding task was run first. The single target presentation task was then run before and after the peripheral crowding task. The performance of patient IG was compared to the averaged control performance using modified paired t-tests designed specifically to test whether single subject's (patient) data fall within the range of control data, using the control group's mean and SD. They provide a robust comparison of a single data point against a small group of controls for single case studies (Crawford and Howell 1998, Crawford and Garthwaite 2002). The performance of all participants was also compared to chance level using unilateral Fisher exact tests. We corrected for multiple comparisons using Bonferroni family-wise error rates.

Experiment 1 - RESULTS

Single target presentation task

As can be seen in Figure 3, IG's accuracy in the letter condition (3B) was very high for all eccentricities (96.67\%) but still lower than controls who performed at ceiling (Crawford test, all p>0.001 all $\mathrm{t}(3)<-$ inf $)$. For color condition only the furthest eccentricity accuracy (76.7\%) was lower than controls (Crawford test $\mathrm{p}<0.01 \mathrm{t}(3)=-11.84)$. Her accuracy, like controls, nevertheless remained largely above chance level at all visual eccentricities (unilateral Fisher exact test, Bonferroni corrected, all p<0.05). In summary, IG was able to identify single color and letter targets in peripheral vision.

Central crowding task 
When the target and distracters were presented at central vision, there were significant differences in performance between IG and the controls. For the color condition (Figure 4A), performance was significantly lower for IG compared to controls with all three spacing $\left(0.8^{\circ}\right.$, $1.5^{\circ}$ and $3^{\circ}$, Crawford test all $\mathrm{p}<0.001 ; \mathrm{t}(3)<-26$ ). In the letter condition (Figure 4B), her performance was significantly worse than controls for the $0.8^{\circ}$ and $1.5^{\circ}$ spacing (Crawford test all $\mathrm{p}<0.01 ; \mathrm{t}(3)<-9.4)$ but was very high and similar to controls for the $3^{\circ}$ spacing (Crawford test $\mathrm{p}>0.1 \mathrm{t}(3)=-0.13$ ). Note however that with both conditions (color and letter), IG's performance remained above chance level at all spacing (unilateral Fisher exact test all $\mathrm{p}<0.05$ ). In summary, when the target was flanked by other colors or letters in central vision, accuracy decreased greatly for patient IG while control performance remained at ceiling.

Increasing stimulus presentation duration did not improve the patient's performance (Figure 4C $\&$ D); performance remained similar even when duration increased from 90 to 110,130 or 150 ms. This reinforces the idea that IG's performance cannot be explained by a deficit of shortterm visual memory (see also Valdois et al. 2019), as at longer durations, the stimuli are onscreen long enough not to require memory to perform the task.

\section{Peripheral crowding task}

Next we investigated performance for three stimuli presented simultaneously in the periphery.

Figure 5 depicts individual participant performance for the three eccentricity conditions $\left(3^{\circ}\right.$ blue, $7^{\circ}$ - red, $10^{\circ}$ - green lines) as a function of target-distracter spacing. The left panel depicts performance in the color conditions, while the right panel depicts performance for the letter conditions. As can be seen in Figures 5A-H, the four age-matched controls showed a classical increase in accuracy with increased target-distracter spacing (e.g., Bouma 1970; Toet \& Levi, 1992; Pelli, Palomares and Majaj 2004), in both letter and color conditions. They also showed asymptoptic performance with increasing eccentricity for a certain target-distracter spacing (e.g., Bouma 1970; Toet and Levi 1992; Latham and Whitaker 1996; Strasburger 2005; Yeshurun and Rashal, 2010). At least 4 spacing by eccentricity were above chance level (unilateral fisher exact test, corrected Bonferroni). In contrast, when the target (color disk or letter) was presented with distracters, the performance of patient IG (Figures 5I and 5H) remained at chance level regardless of target eccentricity and spacing (unilateral Fisher exact test ps<0.05). In summary, IG was unable to correctly identify the flanked target in either the color or letter conditions. 
Experiment 2: A deficit of recognition or of localization?

Tasks

In order to gain insight into the nature of IG's chance level performance for the crowding targetdistracter task, we tested her performance with different instructions, reflecting those of typical visual search tasks. We used the exact same stimuli as in the peripheral crowding task in Experiment 1 with new instructions to simply report the presence or absence of a specific stimulus ( $\mathrm{a}$ in the Letter condition and a «green » disk in the Color condition) within the triplet, regardless of whether it was presented at the first (inner), second (middle) or third (outer) position. This new instruction allowed us to distinguish between three hypotheses. If the performance at chance level of patient IG in the peripheral crowding task reflects an inability to process several elements simultaneously (in apparent contrast with her visual search performance in typical visual displays reported in Khan et al. 2016), she should remain at chance level with this new instruction. If this performance instead reflects increased crowding, her ability to detect the presence of the specified stimulus should be more impaired when it is presented in middle position and should improve with spacing. Finally, if this performance at chance level in the peripheral crowding task reflects a lack of relative position attribution to the three presented stimuli but a simultaneous perception of their identity (which would explain the preservation of pop-out phenomenon in Khan et al. 2016), then patient IG's performance should improve with this new instruction, independently of the target position. In other words, she should be able to detect the presence of the target but not report where it was within the triplet. For this visual search task we used the exact same stimuli as in the peripheral crowding task of Experiment 1 but selected only two large spacing per eccentricity at which we observed negligible crowding impairments in the control subjects:
$\circ$ Eccentricity $3^{\circ}: 2^{\circ}$ and $2.5^{\circ}$
$\circ$ Eccentricity $7^{\circ}: 4^{\circ}$ and $5^{\circ}$
$\circ$ Eccentricity $10^{\circ}: 6^{\circ}$ and $8^{\circ}$

Overall, there was a total of 252 trials each for the letter and color conditions. Patient IG was asked to press the right button when she detected the presence of the specific target within the presented triplet, and the left button when she did not detect any green disk or letter $\mathrm{T}$ (forced choice, chance level was 50\%). 
Following the visual search task, she also performed a single target presentation task with more visual eccentricities $\left(0^{\circ}, 0.5^{\circ}, 1^{\circ}, 2^{\circ}, 3^{\circ}, 4^{\circ}, 5^{\circ}, 6^{\circ}, 7^{\circ}, 10^{\circ}, 11^{\circ}, 12^{\circ}, 16^{\circ}, 18^{\circ}\right)$ in order to obtain a baseline of perceptual accuracy not only for the target's positions but also for the distracters positions (forced choice target identification, chance level was 33.3\%). We also collected the performance of the four age-matched controls in this single target presentation task.

\section{Experiment 2 - Results}

Performance for IG is shown in Figure 6 for the color condition. The presence of the green target was always detected when it was in the inner position regardless of eccentricity. Performance dropped to chance for the outer position at an eccentricity of $7^{\circ}$, and was also not different from chance at $10^{\circ}$, for both the middle and outer positions.

To better understand this pattern of results we analyzed performance as a function of the actual eccentricity of the green target, which varied according to the three tested eccentricities of the triplet $\left(3,7\right.$ and $\left.10^{\circ}\right)$ but also whether the green target was the inner, middle, or outer display item (Figure 7A). This pattern of results is well accounted for by a gradual performance decrease beyond an eccentricity of $10^{\circ}$, irrespective of the target's relative position within the triplet (inner, middle, outer). Note that during the isolated presentation of colored disks (Figure 7b) accuracy similarly decreased to chance level after $10^{\circ}$ of eccentricity except for $12^{\circ}$ (all p>0.05) for IG. However, unlike the single target performance, her performance dropped further below chance level at the furthest eccentricities (Fig. 7A $-16^{\circ} \& 18^{\circ}$ ), suggesting that the patient was "sure" that the green target was not presented in the visual display.

In summary, with color disks, performance in the visual search task can be explained by the decrease of color identification after $10^{\circ}$ of eccentricity even for isolated stimuli presentation. Performance comparison between the peripheral crowding task and the visual search task with the exact same stimuli argues in favor of a localization deficit. Indeed, up to an eccentricity of $10^{\circ}$, the green disk was always detected irrespective of its position within the triplet, whereas when the instruction was to identify the middle target (in the crowding task), performance was at chance level. One can therefore reason that up to $10^{\circ}$ of eccentricity, the three colored disks were seen and perceptually identified but the patient did not know which one was presented at the middle position. 
For the letter condition (Figure 8), IG was unable to detect the presence of the letter T above chance levels ( $p>0.05$ ), except for the inner position (the closest to the fixation point) for triplet eccentricities of $7^{\circ}$ and $10^{\circ}(\mathrm{p}<0.05)$. As in the color condition, we analyzed accuracy as a function of the actual eccentricity at which the target " $T$ " was presented (Figure 9A). While isolated letter identification was at ceiling at all eccentricities (Figure 9B), detecting the presence of the letter " $\mathrm{T}$ " within a triplet was not significantly different from chance level ( $>0.05)$, irrespective of its actual eccentricity, except for the inner position at an eccentricity of $2^{\circ}(\mathrm{p}<0.05)$.

This advantage for the inner position of the triplet (the nearest to the fixation) is confirmed by examining performance as function of triplet visual eccentricity $\left(3,7\right.$ and $\left.10^{\circ}\right)$ and spacing (Figure 10). In sum, with letters, patient IG's ability to perceptually identify one stimulus among three was drastically impaired, irrespective of its actual eccentricity. Identification of the letter "T" was sometimes possible when it was presented at the inner location within the triplet (the nearest to the fixation) but not elsewhere in the triplet, which explains why performance remained at chance level when the instruction was to identify the middle target in the peripheral crowding task.

Experiment 3: Effect of identical distracters

Tasks

The performance of patient IG in the letter condition of Experiment 2 suggests that when letter symbols (but not colored disks) are presented simultaneously, she can only identify one at a time. This still does not explain her performance for feature present/absent search displays where she exhibited a cost (increased search times) to find the target among distracters with respect to normal performance, but this cost remained constant with set size (Khan et al. 2016). To parallel another characteristic of visual search conditions where all distracters were identical, we implemented a perceptual grouping crowding task. We used the same target eccentricities and spacing as in Experiment 2 but the distracters were identical and different from the target, namely two $\mathrm{X}$ letters for the letter condition and two grey disks for the color condition. The instruction was the same as for the peripheral crowding task of Experiment 1: identify the middle target ("red", "blue" or "green" in the color condition and "F", "N"or "T" 
in the letter condition, forced choice, chance level: 1/3). Each of the 3 possible targets was presented 14 times at the 3 peripheral eccentricities and at the 2 largest spacing described for Experiment 2, distributed pseudo-randomly. Overall, there was a total of 252 trials each for the letter and color conditions.

\section{Experiment 3 - Results}

Patient IG's accuracy drastically improved for both letter and color condition with performance above chance level for all conditions of target eccentricity and target-distracter spacing (all $\mathrm{p}<0.05)$ as shown in Figure 11. This figure includes performance with the identical distracters (dashed lines) as well as performance with different distracters from Experiment 1 (solid lines). As can be seen in this figure, perceptual grouping alleviated the major deficit observed in Experiment 1 for the patient.

Thus, in patient IG, grouping the identical distracters improves the extraction of the middle target identity and alleviates the crowding effect. This is in agreement with performance by normal participants reported in the crowding literature (Scolari et al. 2007; Saarela et al. 2009; Freeman et al. 2012; Keshvari and Rosenholtz 2016). Note however that this perceptual grouping may simply resolve the problem of localizing the target; in essence, the patient has a cue that she should report based on the lines that differ from the known distracters (Rosenholtz, $\mathrm{Yu}$ and Keshvari 2019). Indeed, patient IG reported that she clearly saw the target that was different from "X" or "grey" (which allowed her to complete the identification task with a good accuracy), but she was unable to say whether the target was actually in the middle of the triplet. This experiment, therefore, confirms that patient IG's main difficulty is perceiving the spatial relations between several items when presented simultaneously (for letters as well as for colored disks). Each color disk is composed of 1 element, and therefore her deficit affects performance only when the task requires perceiving such relations. But the letters are composed of a spatial configuration of several separable elements, so the identification of each letter by itself already requires understanding such spatial relations. When the stimulus contains only a single letter, the uncertainty about the spatial relations is relatively small and IG manages to perform optimally, likely relying more than controls on prior knowledge (Balas, Nakano and Rosenholtz 2009). But, when there is more than a single letter, there are many more elements and possible spatial relations, and therefore uncertainty considerably increases and accordingly performance is considerably reduced. 
Whether or not this is related to the mechanisms of "crowding" is a difficult question, because the processes underlying crowding are still under debate (for a review, see Rosenholtz et al. 2019); a variety of mechanisms have been proposed, including substitution (Strasburger 2005; van den Berg et al. 2012), spatial receptive field constraints or pooling (Balas, Nakano and Rosenholtz, 2009; Lettvin 1976; Levi 2008; Parkes at al. 2001; Pelli and Tillman 2008), and limits on the resolution of attention (Intriligator and Cavanagh 2001). Regardless of the proposed mechanism, it is clear that crowding can lead to substitution errors, i.e. reporting a distracter instead of the target because information about position is lost or ambiguous, or reporting a mixture of target and distracters lines, as predicted by all of these crowding theories. This appears to resemble IG's deficit. In that regard her deficit may be considered similar to crowding.

\section{DISCUSSION}

Clinical vision reflecting excessive crowding has been previously proposed for amblyopics (Greenwood et al. 2012), dyslexics (Martelli et al. 2009) and patients with occipito-temporal damage (ventral visual stream, Strappini et al. 2017; Sand et al. 2018). The present study explored this hypothesis for patients with damage of the dorsal visual stream by studying in depth the performance of patient IG in crowding tasks consecutive to bilateral superior parietal lobule (SPL) lesions. In this paper, we tested her visual performance with plain objects (blue, red or green colored disks) versus objects consisting of separable lines (letters N, T or F), in conditions of isolated versus simultaneous presentation. In conditions of simultaneous presentation, we contrasted two instructions: identifying the middle target (classical crowding task, Experiment 1) versus detecting the presence of a specific target within the triplet (visual search task, Experiment 2).

For colored disks, simultaneous presentation revealed a relative localization deficit. In an area of up to $10^{\circ}$ of visual eccentricity, patient IG was able to identify whether a green disk was presented within a triplet of colored disks (visual search task) with similar accuracy as when she was required to identify the color (green, red or blue) of a single disk presented at the same location. However, her performance drastically decreased when required to accurately report the color (green, red or blue) of the middle disk of a triplet of colored disks (crowding task). She is therefore able to perceive all three colored disks simultaneously, but she cannot localize them relative to each other. Presentation of grey distracters flanking the middle disk 
(Experiment 3) also allowed her to resolve the localization deficit. When the green target appeared beyond $10^{\circ}$, she was unable to process it, even when presented on its own. This cannot be explained by the decrease of the density of retinal cones (which are involved in color perception) with eccentricity as our four control subjects showed a drop in performance only at larger eccentricities. Note also that performance drops far below chance level in the simultaneous presentation condition in Experiment 2 at the furthest eccentricities, indicating that the patient was convinced that the green target was not present. This suggests that the other stimuli within her attentional field may have captured all attentional resources resulting in an extinction of stimuli positioned outside her attentional field. In sum, IG can process multiple simple colored objects simultaneously if they are presented within her "functional visual field" (inside $10^{\circ}$ of eccentricity), but can localize them relative to each other only in central vision (where her 'crowded' performance was lower than controls but remained above chance level).

For letters, the same dissociated identification (N, T or F) ability was observed at all tested visual eccentricities: performance was normal with isolated presentation but drastically decreased as soon as the letter was presented in the middle of two other target letters. The presentation with two identical X distracters (Experiment 3) alleviated the identification deficit of the middle letter. However, IG could not accurately report the presence of the letter T among the three letters (visual search instruction, Experiment 2), except when the $\mathrm{T}$ was the item closest to fixation.

A unified explanation of the pattern of results observed in this study can be provided, that is, an impaired ability, following bilateral damage of the SPL, to process the relative positions of separable parts (within and between objects). In the case of colored disks, each disk is a simple object, so the impairment loses the location of the disks but does not disrupt their color identification. In the case of letters, even a single letter has multiple simple parts (perhaps the lines that make up the letter). The SPL damage would impair the localization of these parts. The identification of each letter by itself already requires understanding such spatial relations. When the stimulus contains only a single letter, the uncertainty about the spatial relations is relatively small and IG manages to perform optimally (likely relying more than controls on prior knowledge). However, when there is more than a single letter, there are many more elements and possible spatial relations, and therefore uncertainty considerably increases. Accordingly, the localization deficit affects perception to a larger degree than for simple plain objects (such as colored disks). With identical X flankers, the uncertainty is considerably 
reduced: ignoring any diagonals would essentially bring IG back to a state more like a single letter identification.

A deficit of localization and spatial binding of separable parts also explains patient IG's visual search previous results reported in Khan et al. (2016). Detecting the presence or absence of a specific object (a red disk) among others (e.g. red squares and green disks) does not require its precise localization, and object's color and shape are not spatially separable features. The fact that the localization deficit did not affect perception of simultaneous colored disks is consistent with the preservation of pop-out and conjunction visual searches for colored shapes (Khan et al. 2016). The present results showing a localization deficit affecting perception for simultaneous presentation of objects made by spatially separable lines (here letters) also fits with the impairment of feature-present and feature-absent searches (Khan et al. 2016).

Note that acuity, binocular vision, temporal threshold for single letter identification, and delayed multi-letter report with sequential presentation have been reported to be fully normal in patient IG (Valdois et al. 2019). We also showed here that increasing presentation time did not allow her to improve identification performance in the condition of simultaneous presentation in central vision (Experiment 1). Her deficit can therefore be explained neither by a reduced visual processing speed nor by reduced visual short-term memory capacity (unlike other patients with developmental dyslexia or simultanagnosia consecutive to neurodegenerative disease reported in the literature (Neitzel et al 2016; Bogon et al. 2014). Her deficit therefore lies in the spatial rather than in the temporal domain.

Whether objects consist of separable lines or not appears as an important factor for the limitation of simultaneous object processing consecutive to SPL dysfunction, suggesting an inability to establish spatial relations between the different elements and to automatically attribute them to one object or another. One interpretation might be that patient IG presents an impairment at the level of perceptual organization processes that structure the bits and pieces of visual information into larger entities that correspond to meaningful objects (see Kimchi et al. 2007; Yeshurun et al. 2009). This would predict poor object individuation; such individuation necessarily precedes identification of multiple complex objects (Xu and Chun 2009), but might not be required for simpler objects (Mazza 2017). Nevertheless, some perceptual organization processes are preserved, since patient IG was clearly able to make use of and perhaps automatically group the features of the identical distracters "X", improving (but not fully restoring) the identification of the middle target in Experiment 3. 
Another interpretation could be excessive crowding. Similarity effects, in which the target is easier to report when flanked by distracters that group with each other and not with the target, abound in crowding (Livne and Sagi, 2007; Saarela et al. 2009; Manassi et al. 2012; Freeman et al. 2012; Keshvari and Rosenholtz 2016). A popular explanation of crowding known as the texture tiling model can explain a number of these effects (Rosenholtz, Yu and Keshvari 2019) but not all of them (Doerig et al. 2019). This pooling model represents the peripheral input in terms of a rich set of summary image statistics, e.g. correlations between responses of V1-like "cells". The pooling regions "pool" or summarize the statistics over an area that grows (in the normal human visual system) linearly with eccentricity. These rich summary representations capture a great deal of useful information at the expense of precise localization of the features, leading to misreporting one of the distracters instead of the target. To get intuitions of what the model predicts, one can generate visualizations of the information preserved and lost according to the model (Balas et al. 2009). Examining these visualizations, it appears that the summary statistics can better represent a target letter when flanked by identical letters (Keshvari and Rosenholtz 2016; Rosenholtz et al. 2019). Simple shapes like disks of a single color "cohere", though their location may be ambiguous, whereas in these visualizations more complex shapes like letters can split into pieces and re-"tile" into novel shapes, leading to difficult recognition and hard search for, e.g. an $\mathrm{O}$ among Qs (Chang and Rosenholtz, 2016). However, IG's performance also deviates significantly from normal crowding. IG shows crowding in the fovea, where pooling regions are presumed to be small (Experiment 1). In the periphery there was no sign of a critical spacing at which crowding is relieved (Experiment 1). If the pooling regions are fixed, perhaps corresponding to receptive fields, it seems implausible that IG's SPL damage causes pooling regions to grow larger in both central and peripheral vision.

Alternatively, the SPL is usually acknowledged for its role in endogenous attention, and in patient IG endogenous cueing has shorter effect than controls in a Posner task (Valdois et al. 2019). Given the connections described above between IGs performance and signatures of crowding, it is worth noting that attention can modulate crowding performance in the sense that observers are better at recognizing a crowded object when a pre-cue indicates the target location in advance. Specifically, both predictive and non-predictive peripheral cues (e.g., Rashal and Yeshurun 2014; Strasburger and Malania 2013; Yeshurun and Rashal 2010) as well as predictive central cues (e.g., Felisberti, Solomon and Morgan 2005), that allowed advanced allocation of attention to the target location, resulted in reduced critical spacing. It has also been shown with central targets that focusing attention reduced the critical distance (Albonico et al. 
2018). If attention indeed reduces the critical spacing, and assuming that normal observers allocate some amount of attention to peripheral targets thereby reducing the critical spacing, then with a lesion that impairs attention, one might end up with a bigger critical spacing. Attention is also considered to be required to apprehend spatial relations between items (Logan 1994; Poder 1999; Moore et al 2001) and to bind together a within-object structure (the overall shape of an object is not available pre-attentively, Wolfe and Bennett 1997). Patient IG has a deficit specific to spatial binding of separable lines, her ability to bind color and shape features is fully preserved, as she is as fast as controls in conjunction search (Khan et al. 2016). Therefore, the deficit consecutive to the bilateral SPL damage (visual dorsal stream) may lie at the expected level of spatial attention, with effects on object perception and crowding.

Damage to the SPL has repeatedly been shown to produce visual localisation deficits (Mishkin and Ungerleider 1982; Perenin \& Vighetto 1988; Milner and Goodale 1993, 2008; Blangero et al. 2010) but defining the level of functional impairment helps in clarifying the debated role of the dorsal visual stream ("How" versus "Where"). The predominant dual-stream model of visual processing (Milner and Goodale 1993, 2008) put forward that the dorsal visual stream is devoted to the « vision for action » (« How »), while « vision for perception » would be entirely based on the ventral visual stream (infero-temporal cortex). It has highlighted the errors reported in numerous patients with unilateral SPL lesions when pointing toward visual targets (optic ataxia; see for example Perenin \& Vighetto 1988 and Blangero et al. 2010). Mishkin and Ungerleider (1982) previously proposed a more general « Where » function for the dorsal visual stream, showing that its lesion impaired the ability to perform the perceptual landmark test (midline judgement). Our present result further demonstrates that the dorsal visual stream contributes to both visually-guided action and visuo-perceptual performance, and that localisation deficits («where» function) can affect both, especially in patients with bilateral damage. This localization deficit does not correspond to excessive perceptual crowding but affects simultaneous object identification by increasing spatial uncertainty.

\section{ACKNOWLEDGEMENTS}

The authors would like to thank Romeo Salemme for technical assistance. LP was supported by the CNRS and the Labex/Idex ANR-11-LABX-0042 (France). AV was supported by the Fondation de France. 
Figure legends

Figure 1. Horizontal (axial) T2-weighted magnetic resonance section of patient IG's brain (at $\mathrm{z}$ coordinate $=85$ in standard Talairach space) showing fairly symmetric bilateral lesion involving mainly the intraparietal sulcus (IPS, dotted line) and Brodmann's area 7 in the superior parietal lobule (SPL). LH: left hemisphere. RH: right hemisphere.

Figure 2. Trial sequence. Each trial began with a central fixation cross for $400 \mathrm{~ms}$ on which the participant was asked to fixate. Next the stimuli (in this case the 3 colored disks for the central vision colored condition) were presented briefly for $90 \mathrm{~ms}$. This was followed by the reappearance of the central fixation dot that remained until the participant entered a response.

Figure 3. Single target presentation conditions. Performance for IG (dotted black line and dots) and controls (gray line and dots) are shown for the color (A) and letter (B) conditions as a function of target eccentricity. The y-axis (range 50 to 100\%) represents accuracy in correctly identifying the color or letter presented. $*=p<0.05$. Error bars are standard deviations across control participants.

Figure 4. Central crowding task. Performance for IG (dotted black line and dots) and controls (gray line and dots) are shown for the color (A) and letter (B) conditions as a function of the spacing between the target and the distracters. The y-axis (50-100\%) represents accuracy in correctly identifying the color or letter presented. $*=p<0.05$. Error bars are standard deviations across control participants.

Figure 5. Peripheral crowding task. Performance for each individual participant is shown for the color (left panel) and the letter (right panel) conditions. Accuracy as a function of spacing is shown for each of the three target eccentricities $\left(3^{\circ}\right.$ - light grey dotdashed line and dots, $7^{\circ}$ grey dashed line and dots, $10^{\circ}$ - black dotted line and dots). Solid dots depict performance that is significantly different from chance (horizontal tight dotted line $-33 \%$ ), while open dots depict performance that is not significantly different from chance. Each row depicts performance for each individual participant, where $\mathbf{A}-\mathbf{H}$ are control performance and $\mathbf{I}$ and $\mathbf{J}$ are IG's results. The y-axis (20-100\%) represents accuracy in identifying the color or letter presented.

Figure 6. Visual search instruction for peripheral crowding color task. Performance for IG is shown for the color condition for the three target eccentricities $\left(3^{\circ}\right.$ - light grey dotdashed line and dots, $7^{\circ}$ - grey dashed line and dots, $10^{\circ}$ - black dotted line and dots). Solid dots depict 
performance that is significantly different from chance (horizontal tight dotted line $-50 \%$ ), while open dots depict performance that is not significantly different from chance. The y-axis (0-100\%) represents accuracy in responding whether or not the target (green disk) was present.

Figure 7. Eccentricity vs. performance for visual search instruction color task. A. Performance for IG as a function of actual visual eccentricity of the target separated by position, inner (blue line and dots), middle (red line and dots) and outer (green line and dots). B. Performance for the single target presentation with the visual search instruction for IG (black dotted line and dots) and controls (gray line and dots with SD error bars across controls). Solid dots depict performance that is significantly different from chance (horizontal tight dotted line $-50 \%$ ), while open dots depict performance that is not significantly different from chance. The $\mathrm{y}$-axis $(0-100 \%)$ represents accuracy in responding whether or not the target (green disk) was present.

Figure 8. Visual search instruction for peripheral crowding letter task. Performance for IG is shown for the letter condition for the three target eccentricities $\left(3^{\circ}\right.$ - light grey dotdashed line and dots, $7^{\circ}$ - grey dashed line and dots, $10^{\circ}$ - black dotted line and dots). Solid dots depict performance that is significantly different from chance (horizontal tight dotted line $-50 \%$ ), while open dots depict performance that is not significantly different from chance. The y-axis (0-100\%) represents accuracy in responding whether or not the target (letter T) was present.

Figure 9. Eccentricity vs. performance for visual search instruction letter task. A. Performance for IG is shown as a function of actual visual eccentricity of the target separated by position, inner (blue line and dots), middle (red line and dots) and outer (green line and dots). B. Performance for the single target presentation with the visual search instruction for IG (black dotted line and dots) and controls (gray line and dots with SD error bars across controls). Solid dots depict performance that is significantly different from chance (horizontal tight dotted line $-50 \%)$, while open dots depict performance that is not significantly different from chance. The y-axis $(0-100 \%)$ represents accuracy in responding whether or not the target (green disk) was present.

Figure 10. Visual search instruction for peripheral crowding letter task as a function of spacing and position. Performance for IG is shown as a function of spacing and eccentricity of the target separated by position, inner (blue line and dots), middle (red line and dots) and outer (green line and dots). Solid dots depict performance that is significantly different from chance (horizontal tight dotted line $-50 \%$ ), while open dots depict performance that is not 
significantly different from chance. The y-axis (0-100\%) represents accuracy in responding whether or not the target (letter T) was present.

Figure 11. Perceptual grouping task. Patient IG's performance is shown for the perceptual grouping task (identical distracters) for the color (A) and the letter (B) conditions. For comparison, performance from the peripheral crowding task from experiment 1 is reproduced here from figure $\mathbf{5 I}$ and $\mathbf{J}$. The perceptual grouping performance is shown for the same three target eccentricities with the same three grey levels but with solid lines. Solid dots depict performance that is significantly different from chance (horizontal tight dotted line $-33 \%$ ), while open dots depict performance that is not significantly different from chance. The y-axis (range 20 to 100\%) represents accuracy in correctly identifying the color or letter presented. 


\section{Bibliography}

Albonico A, Martelli M, Bricolo E, Frasson E, Daini R. 2018. Focusing and orienting spatial attention differently modulate crowding in central and peripheral vision. J Vis. 18(3):4. doi:10.1167/18.3.4.

Balas B, Nakano L, Rosenholtz R. 2009. A summary-statistic representation in peripheral vision explains visual crowding. J Vis. 9(12):13.1-18. doi:10.1167/9.12.13.

van den Berg R, Johnson A, Martinez Anton A, Schepers AL, Cornelissen FW. 2012. Comparing crowding in human and ideal observers. J Vis. 12(6):13. doi:10.1167/12.6.13.

Blangero A, Ota H, Rossetti Y, Fujii T, Luaute J, Boisson D, Ohtake H, Tabuchi M, Vighetto A, Yamadori A, Vindras P, Pisella L. 2010. Systematic retinotopic error vectors in unilateral optic ataxia. Cortex. 46(1): 77-93. doi: 10.1016/j.cortex.2009.02.015.

Bogon J, Finke K, Schulte-Körne G, Müller HJ, Schneider WX, Stenneken P. 2014. Parameterbased assessment of disturbed and intact components of visual attention in children with developmental dyslexia. Dev Sci. 17(5):697-713. doi:10.1111/desc.12150.

Bosse M-L, Tainturier MJ, Valdois S. 2007. Developmental dyslexia: the visual attention span deficit hypothesis. Cognition. 104(2):198-230. doi:10.1016/j.cognition.2006.05.009.

Bouma H. 1970. Interaction effects in parafoveal letter recognition. Nature. 226(5241):177178. doi:10.1038/226177a0.

Casco C, Prunetti E. 1996. Visual search of good and poor readers: effects with targets having single and combined features. Percept Mot Skills. 82(3 Pt 2):1155-1167. doi:10.2466/pms.1996.82.3c.1155.

Chang H, Rosenholtz R. 2016. Search performance is better predicted by tileability than presence of a unique basic feature. J Vis. 16(10):13. doi:10.1167/16.10.13.

Chechlacz M, Gillebert CR, Vangkilde SA, Petersen A, Humphreys GW. 2015. Structural Variability within Frontoparietal Networks and Individual Differences in Attentional Functions: An Approach Using the Theory of Visual Attention. J Neurosci. 35(30):1064710658. doi:10.1523/JNEUROSCI.0210-15.2015. 
Crawford JR, Garthwaite PH. 2002. Investigation of the single case in neuropsychology: confidence limits on the abnormality of test scores and test score differences. Neuropsychologia. 40(8):1196-1208. doi:10.1016/s0028-3932(01)00224-x.

Crawford JR, Howell DC. 1998. Comparing an Individual's Test Score Against Norms Derived from Small Samples. The Clinical Neuropsychologist. 12(4):482-486. doi:10.1076/clin.12.4.482.7241.

Demeyere N, Rzeskiewicz A, Humphreys KA, Humphreys GW. 2008. Automatic statistical processing of visual properties in simultanagnosia. Neuropsychologia. 46(11):2861-2864. doi:10.1016/j.neuropsychologia.2008.05.014.

Doerig A, Bornet A, Rosenholtz R, Francis G, Clarke AM, Herzog MH. 2019. Beyond Bouma's window: How to explain global aspects of crowding? PLoS Comput Biol. 15(5):e1006580. doi:10.1371/journal.pcbi.1006580.

Felisbert FM, Solomon JA, Morgan MJ. 2005. The role of target salience in crowding. Perception. 34(7):823-833. doi:10.1068/p5206.

Freeman J, Chakravarthi R, Pelli DG. 2012. Substitution and pooling in crowding. Atten Percept Psychophys. 74(2):379-396. doi:10.3758/s13414-011-0229-0.

Greenwood JA, Tailor VK, Sloper JJ, Simmers AJ, Bex PJ, Dakin SC. 2012. Visual acuity, crowding, and stereo-vision are linked in children with and without amblyopia. Invest Ophthalmol Vis Sci. 53(12):7655-7665. doi:10.1167/iovs.12-10313.

Hulleman J, Olivers CNL. 2017. The impending demise of the item in visual search. Behav Brain Sci. 40:e132. doi:10.1017/S0140525X15002794.

Intriligator J, Cavanagh P. 2001. The spatial resolution of visual attention. Cogn Psychol. 43(3):171-216. doi:10.1006/cogp.2001.0755.

Jackson GM, Shepherd T, Mueller SC, Husain M, Jackson SR. 2006. Dorsal simultanagnosia: An impairment of visual processing or visual awareness? Cortex. 42(5):740-749. doi:10.1016/s0010-9452(08)70412-x.

Keshvari S, Rosenholtz R. 2016. Pooling of continuous features provides a unifying account of crowding. J Vis. 16(3):39. doi:10.1167/16.3.39. 
Khan AZ, Prost-Lefebvre M, Salemme R, Blohm G, Rossetti Y, Tilikete C, Pisella L. 2016. The Attentional Fields of Visual Search in Simultanagnosia and Healthy Individuals: How Object and Space Attention Interact. Cereb Cortex. 26(3):1242-1254. doi:10.1093/cercor/bhv059.

Kimchi R, Yeshurun Y, Cohen-Savransky A. 2007. Automatic, stimulus-driven attentional capture by objecthood. Psychon Bull Rev. 14(1):166-172. doi:10.3758/bf03194045.

Latham K, Whitaker D. 1996. Relative roles of resolution and spatial interference in foveal and peripheral vision. Ophthalmic Physiol Opt. 16(1):49-57.

Lettvin JY. 1976. On Seeing Sidelong. The Sciences. 16(4):10-20. doi:10.1002/j.23261951.1976.tb01231.x.

Levi DM. 2008. Crowding--an essential bottleneck for object recognition: a mini-review. Vision Res. 48(5):635-654. doi:10.1016/j.visres.2007.12.009.

Livne T, Sagi D. 2007. Configuration influence on crowding. J Vis. 7(2):4.1-12. doi:10.1167/7.2.4.

Lobier M, Zoubrinetzky R, Valdois S. 2012. The visual attention span deficit in dyslexia is visual and not verbal. Cortex. 48(6):768-773. doi:10.1016/j.cortex.2011.09.003.

Logan GD. 1994. Spatial attention and the apprehension of spatial relations. J Exp Psychol Hum Percept Perform. 20(5):1015-1036. doi:10.1037//0096-1523.20.5.1015.

Luria AR. 1959. Disorders of "simultaneous perception" in a case of bilateral occipito-parietal brain injury. Brain. 82:437-449. doi:10.1093/brain/82.3.437.

Manassi M, Sayim B, Herzog MH. 2012. Grouping, pooling, and when bigger is better in visual crowding. J Vis. 12(10):13. doi:10.1167/12.10.13.

Martelli M, Di Filippo G, Spinelli D, Zoccolotti P. 2009. Crowding, reading, and developmental dyslexia. J Vis. 9(4):14.1-18. doi:10.1167/9.4.14.

Mazza V. 2017. Simultanagnosia and object individuation. Cogn Neuropsychol. 34(7-8):430439. doi:10.1080/02643294.2017.1331212.

Milner AD, Goodale MA. 1993. Visual pathways to perception and action. In: Hicks TP, Molotchnikoff S, Ono T (eds) Progress in brain research, vol. 95. The visually responsive 
neuron: from basic neurophysiology to behaviour. Elsevier, Amsterdam, pp 317-337. doi:10.1016/S0079-6123(08)60379-9

Milner AD, Goodale MA. 2008. Two visual systems re-viewed. Neuropsychologia 46:774785. doi:10.1016/j.neuropsychologia.2007.10.005

Mishkin M, Ungerleider LG. 1982 Contribution of striate inputs to the visuospatial functions of parieto-preoccipital cortex in monkeys. Behav Brain Res. 6(1):57-77. doi: 10.1016/01664328(82)90081-x.

Moore CM, Elsinger CL, Lleras A. 2001. Visual attention and the apprehension of spatial relations: the case of depth. Percept Psychophys. 63(4):595-606. doi:10.3758/bf03194424.

Neitzel J, Ortner M, Haupt M, Redel P, Grimmer T, Yakushev I, Drzezga A, Bublak P, Preul C, Sorg C, et al. 2016. Neuro-cognitive mechanisms of simultanagnosia in patients with posterior cortical atrophy. Brain. 139(Pt 12):3267-3280. doi:10.1093/brain/aww235.

Parkes L, Lund J, Angelucci A, Solomon JA, Morgan M. 2001. Compulsory averaging of crowded orientation signals in human vision. Nat Neurosci. 4(7):739-744. doi:10.1038/89532.

Pelli DG, Palomares M, Majaj NJ. 2004. Crowding is unlike ordinary masking: distinguishing feature integration from detection. J Vis. 4(12):1136-1169. doi:10.1167/4.12.12.

Pelli DG, Tillman KA. 2008. The uncrowded window of object recognition. Nat Neurosci. 11(10):1129-1135. doi:10.1038/nn.2187.

Perenin MT, Vighetto A. 1988. Optic ataxia: a specific disruption in visuomotor mechanisms. I. Different aspects of the deficit in reaching for objects. Brain 111 ( Pt 3):643-74. doi: 10.1093/brain/111.3.643.

Peyrin C, Démonet JF, N'Guyen-Morel MA, Le Bas JF, Valdois S. 2011. Superior parietal lobule dysfunction in a homogeneous group of dyslexic children with a visual attention span disorder. Brain Lang. 118(3):128-138. doi:10.1016/j.bandl.2010.06.005.

Põder E. 1999. Search for feature and for relative position: measurement of capacity limitations. Vision Res. 39(7):1321-1327. doi:10.1016/s0042-6989(98)00253-3.

Rashal E, Yeshurun Y. 2014. Contrast dissimilarity effects on crowding are not simply another case of target saliency. Journal of Vision. 14(6):9-9. doi:10.1167/14.6.9. 
Reilhac C, Peyrin C, Démonet J-F, Valdois S. 2013. Role of the superior parietal lobules in letter-identity processing within strings: FMRI evidence from skilled and dyslexic readers. Neuropsychologia. 51(4):601-612. doi:10.1016/j.neuropsychologia.2012.12.010.

Rosenholtz R, Huang J, Raj A, Balas BJ, Ilie L. 2012. A summary statistic representation in peripheral vision explains visual search. J Vis. 12(4). doi:10.1167/12.4.14.

Rosenholtz R, Yu D, Keshvari S. 2019. Challenges to pooling models of crowding: Implications for visual mechanisms. J Vis. 19(7):15. doi:10.1167/19.7.15.

Saarela TP, Sayim B, Westheimer G, Herzog MH. 2009. Global stimulus configuration modulates crowding. J Vis. 9(2):5.1-11. doi:10.1167/9.2.5.

Sand K, Robotham RJ, Martelli M, Starrfelt R. 2018. Visual crowding in pure alexia and $\begin{array}{llll}\text { acquired } & \text { prosopagnosia. } & \text { 35(7):361-370. }\end{array}$ doi:10.1080/02643294.2018.1483325.

Scolari M, Kohnen A, Barton B, Awh E. 2007. Spatial attention, preview, and popout: which factors influence critical spacing in crowded displays? J Vis. 7(2):7.1-23. doi:10.1167/7.2.7.

Strappini F, Pelli DG, Di Pace E, Martelli M. 2017. Agnosic vision is like peripheral vision, which is limited by crowding. Cortex. 89:135-155. doi:10.1016/j.cortex.2017.01.012.

Strasburger H. 2005. Unfocused spatial attention underlies the crowding effect in indirect form vision. J Vis. 5(11):1024-1037. doi:10.1167/5.11.8.

Thomas C, Kveraga K, Huberle E, Karnath H-O, Bar M. 2012. Enabling global processing in simultanagnosia by psychophysical biasing of visual pathways. Brain. 135(Pt 5):1578-1585. doi:10.1093/brain/aws066.

Toet A, Levi DM. 1992. The two-dimensional shape of spatial interaction zones in the parafovea. Vision Res. 32(7):1349-1357. doi:10.1016/0042-6989(92)90227-a.

Valdois S, Lassus-Sangosse D, Lallier M, Moreaud O, Pisella L. 2019. What bilateral damage of the superior parietal lobes tells us about visual attention disorders in developmental dyslexia. Neuropsychologia. 130:78-91. doi:10.1016/j.neuropsychologia.2018.08.001. 
Whitney D, Levi DM. 2011. Visual crowding: a fundamental limit on conscious perception and object recognition. Trends Cogn Sci (Regul Ed). 15(4):160-168. doi:10.1016/j.tics.2011.02.005.

Wolfe JM, Bennett SC. 1997. Preattentive object files: shapeless bundles of basic features. Vision Res. 37(1):25-43. doi:10.1016/s0042-6989(96)00111-3.

Wolpert I. 1924. Die simultanagnosie — störung der gesamtauffassung. Z f d g Neur u Psych. 93(1):397-415. doi:10.1007/BF02900065.

$\mathrm{Xu}$ Y, Chun MM. 2009. Selecting and perceiving multiple visual objects. Trends Cogn Sci (Regul Ed). 13(4):167-174. doi:10.1016/j.tics.2009.01.008.

Yeshurun Y, Kimchi R, Sha'shoua G, Carmel T. 2009. Perceptual objects capture attention. Vision Res. 49(10):1329-1335. doi:10.1016/j.visres.2008.01.014.

Yeshurun Y, Rashal E. 2010. Precueing attention to the target location diminishes crowding and reduces the critical distance. J Vis. 10(10):16. doi:10.1167/10.10.16. 